Rapid Communications

\title{
Direct Lipido-Metabolomics of Single Floating Cells for Analysis of Circulating Tumor Cells by Live Single-cell Mass Spectrometry
}

\author{
Eiso Hiyama, ${ }^{* \dagger}$ Ahmed Ali, $* * * * *$ Sara Amer, $* * * * *$ Takanori Harada,* Kazumi Shimamoto,* \\ Rie Furushima, $* * *$ Yasmine Abouleila, $* *, * * *$ Samy Emara, $* *$ and Tsutomu MasujIma $* * * \dagger$ \\ *Natural Science Center for Basic Research and Development, Hiroshima University, 1-2-3, Kasumi, \\ Minami, Hiroshima 734-8551, Japan \\ **Faculty of Pharmacy, Misr International University, Km28 Ismailia Road, Cairo, Egypt \\ ***Quantitative Biology Center (QBiC), RIKEN, 6-2-3 Furuedai, Suita, Osaka 565-0874, Japan
}

\begin{abstract}
Direct trapping of a single floating cell, i.e. a white blood cell from a drop of blood, within a nanospray tip was followed by super-sonication after the addition of ionization solvent. Molecular detection of an increased number of peaks with a higher intensity and a wider $\mathrm{m} / \mathrm{z}$ range, which extends from metabolites to lipids, was acquired than of that without sonication. This method was applied to a few separated circulating tumor cells (CTC) from a neuroblastoma patient's blood to obtain their lipido-metabolomic molecular profile at the single cell level. In addition to vital molecules such as amino acids, catechol amine metabolites, which are specific to neuroblastoma, and drugs included in the patient's course of therapy were detected. This established "direct single-cell lipido-metabolomic method" seems to be useful for direct and wide range molecular detection not only for many live single-cells, but also for rare cells, such as CTCs, for future molecular diagnosis.
\end{abstract}

Keywords Live single-cell mass spectrometry, circulating tumor cell, neuroblastoma, floating cells, lipido-metabolomics, one-drop blood analysis

(Received November 16, 2015; Accepted November 24, 2015; Published December 10, 2015)

Blood is essential for life and consists of both biological fluid and blood cells. In the event of disease, afflicted organs might leak molecules and abnormal cells such as tumor cells into the bloodstream. Circulating tumor cells (CTC) are a recently developing target for "liquid biopsy" 1,2 which is often carried out by only counting few CTCs number to diagnose cancer and the stage of disease. ${ }^{3}$ Although these cells have been analyzed by morphology, surface molecular markers, or the combination of both by flow cytometry, ${ }^{4,5}$ detailed molecular aspects, especially in metabolites and lipids, have not been revealed for such few number of cells in blood. If CTCs can be isolated and analyzed at the single-cell level, evaluating the heterogeneity of tumor cells and selecting drugs for molecular target therapy will be possible without surgical resection of the tumors. ${ }^{6}$ Moreover, CTCs can be used for treatment evaluation by monitoring both their count $^{7}$ and intracellular metabolites as well as early detection of relapses. ${ }^{8}$

Live single-cell mass spectrometry ${ }^{9-12}$ seems to be a promising method to resolve previously mentioned problems, however, this method has been mainly applied to adhesive cells because the contents of such cells are more easily sucked by a nanospray tip. This method has also focused mainly on a small molecular range, from $\mathrm{m} / \mathrm{z} 100$ to 500 , where many metabolites can be found. If we can directly suck a whole floating cell into a nanospray tip, effectively homogenize it in the tip at the least dilution, and feed all the contents into a mass spectrometer, including its lipids, which are in higher molecular range of $\mathrm{m} / \mathrm{z}$

$\dagger$ To whom correspondence should be addressed. E-mail: eiso@hiroshima-u.ac.jp (E. H.) (clinical); masujima@ riken.jp (T. M.) (analytical)
$300-1000$, it is possible to accomplish comprehensive and wide molecular range detection of many types of dynamically changing floating cells, including circulating blood cells. This method will be very useful for future diagnosis.

In this paper, we first worked to trap floating white blood cells (WBC) from a single drop of healthy human blood. Then we investigated efficient homogenization of the trapped cells using supersonic waves to get a wider range of molecular peaks in the single-cell mass spectrum. After establishment of this method, we applied it to isolated CTCs from a cancer patient.

WBC preparation: A blood lancet was used to acquire a single drop of blood from the finger of a healthy male adult individual. $5 \mu \mathrm{L}$ of the blood were diluted with $500 \mu \mathrm{L}$ of saline solution $(0.09 \%)$ for a stock solution. From this, $100 \mu \mathrm{L}$ were added to a $35-\mathrm{mm}$ plastic cell culture dish containing $500 \mu \mathrm{L}$ of saline. As shown in Fig. 1, a single WBC was chosen under microscope (only lymphocytes were selected by morphology) and sucked into a nanospray tip (CT-1, Humanix, Japan) using a micromanipulator and piston syringe. A $2-\mu \mathrm{L}$ volume of organic solvent (80\% methanol, 10\% dimethyl sulfoxide (DMSO), and $0.1 \%$ formic acid (for positive mode) or $10 \mathrm{mmol}$ ammonium formate (for negative mode)) was introduced into the nanospray needle from the rear end. Then super-sonication was carried out for $5 \mathrm{~s}$ by suspending the end of the homogenizer rod in the fixed Eppendorf tube.

CTC preparation: As shown in Fig. 2, a 5-mL blood sample from a patient suspected to have neuroblastoma was collected in tubes containing ethylenediaminetetraacetic acid (EDTA). CTC enrichment was achieved by density gradient centrifugation using a Leucosep ${ }^{\mathrm{TM}}$ tube (Greiner Bio-One, Austria) with Ficoll-PaquePlus separation medium (Greiner Bio-One, Austria), 
performed in accordance with the manufacturer's instructions. Collected mononuclear cells were stained with fluorescent antibodies, APC mouse anti-human CD45 (BD 560973) and PE mouse anti-human disaloganglioside GD2 (BD 562100). The negative CD45 and positive GD2 cells were sorted by flow cytometry (BD, Sorp ARIA).

Mass spectrometry: Molecular detection of the isolated WBCs and CTCs was carried out using a high resolution mass spectrometer (LTQ-Orbitrap Velos Pro, Thermo Fisher Scientific) equipped with a nanospray ionization source. The spray voltage was applied around $1-1.5$ or -0.8 to $-1 \mathrm{kV}$, and
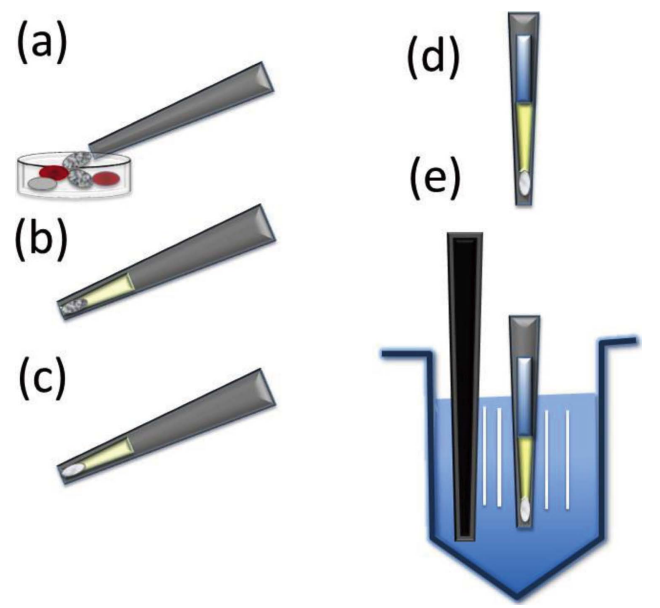

Fig. 1 Capturing of a single WBC under microscope into a nanospray tip using a micro-manipulator and piston syringe. (a) Nanospray tip with positive inner pressure approaching the target cell. (b) The inner pressure was released and the target cell was sucked into the tip. (c) Tip containing a single cell was frozen for stock of samples. (d) Ionization solvent was introduced to the back end of the tip. (e) The front end of the tip was placed in an Eppendorf tube (Eppendorf, Germany) containing the same ionization organic solvent for super sonication. Afterwards, the trapped contents of the tip was fed into the mass spectrometer. the measuring $\mathrm{m} / \mathrm{z}$, range was set to 100 to 1000 , divided into increments of 50 . Raw data was analyzed by MarkerView (Sciex) software in order to perform the peak alignment of the obtained spectra and the paired $t$-test analysis. Detected peaks were estimated by the exact $\mathrm{m} / \mathrm{z}$ value of $\pm 3 \mathrm{ppm}$ tolerance, and annotated by KEGG database (http://www.genome.jp/kegg/ kegg_ja.html) and LIPID MAPS (http://www.lipidmaps.org/).

\section{Results and Discussion}

The spectra of WBCs with super-sonication showed more peaks than without sonication, especially in the mass range higher than 300 where lipids can be found, as shown in Figs. 3(a) and 3(b). The number of peaks which matched to the Human Metabolome Data Base (http://www.hmdb.ca/) was 216 for sonicated cells and only 12 for cells without sonication. This shows that sonication enables us to detect not only metabolites but also lipids at the same time. We found many essential
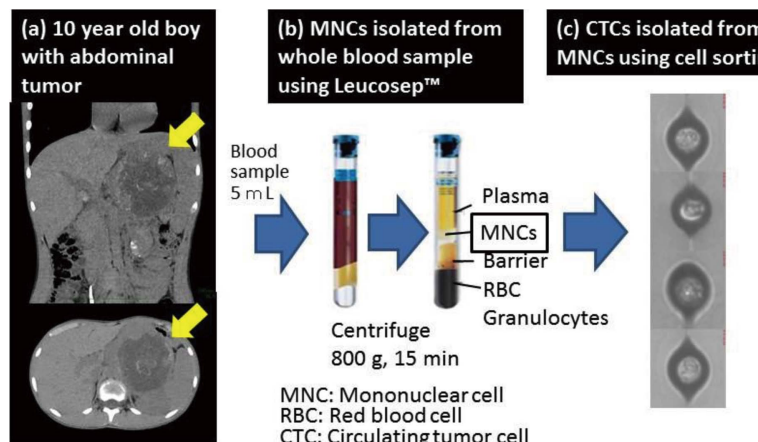
MNCs using cell sorting using Leucosep ${ }^{\text {TM }}$
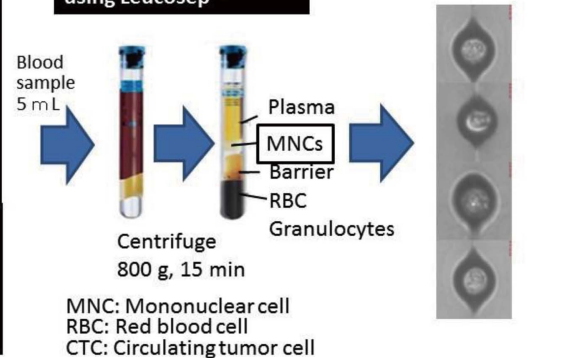

Fig. 2 Isolation of a single CTC. (a) Neuroblastoma case from a 10 year old boy: CT scan revealed a large left abdominal tumor (yellow arrows). Consequently, this tumor was diagnosed as neuroblastoma by tumor biopsy. (b) Before biopsy, a 5-mL blood sample from this patient was collected and mononuclear cells (MNCs) were isolated by density gradient centrifugation using a Leucosep ${ }^{\mathrm{TM}}$ tube. (c) CTCs were isolated as negative CD45 and positive GD2 cells using flow cytometry.

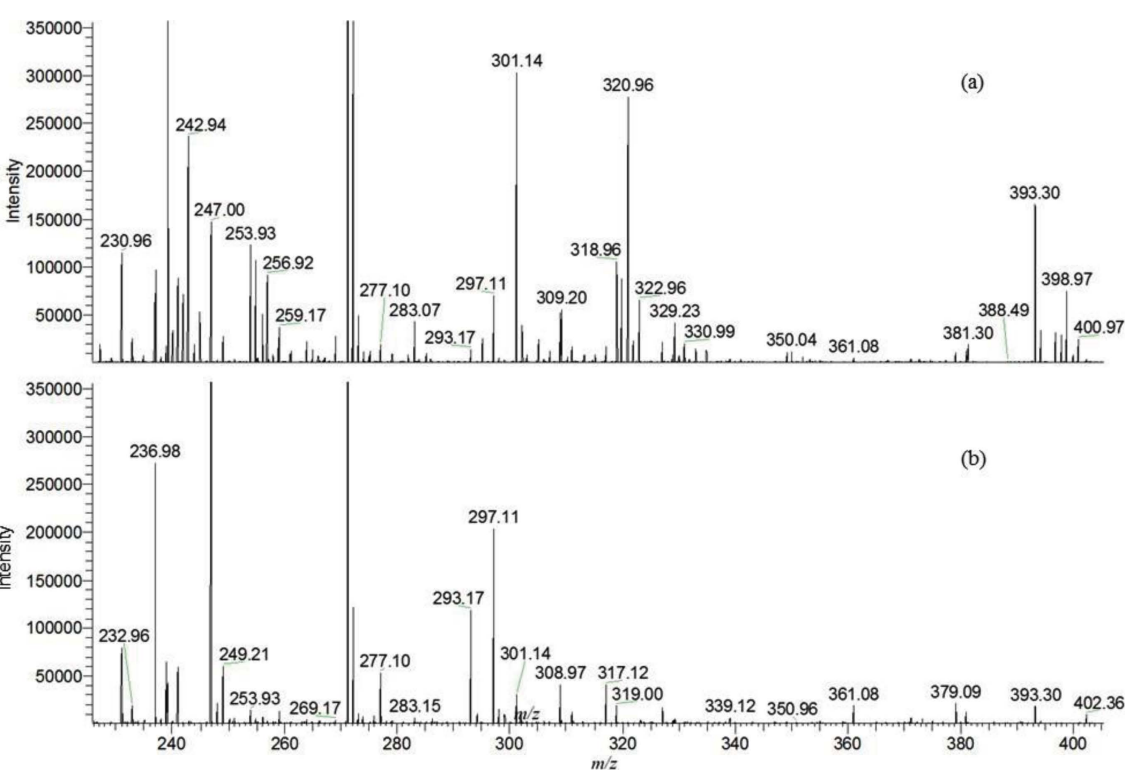

Fig. 3 Mass spectra showing metabolite and lipid peaks from a WBC (a) with sonication, (b) without sonication in positive ion mode. 


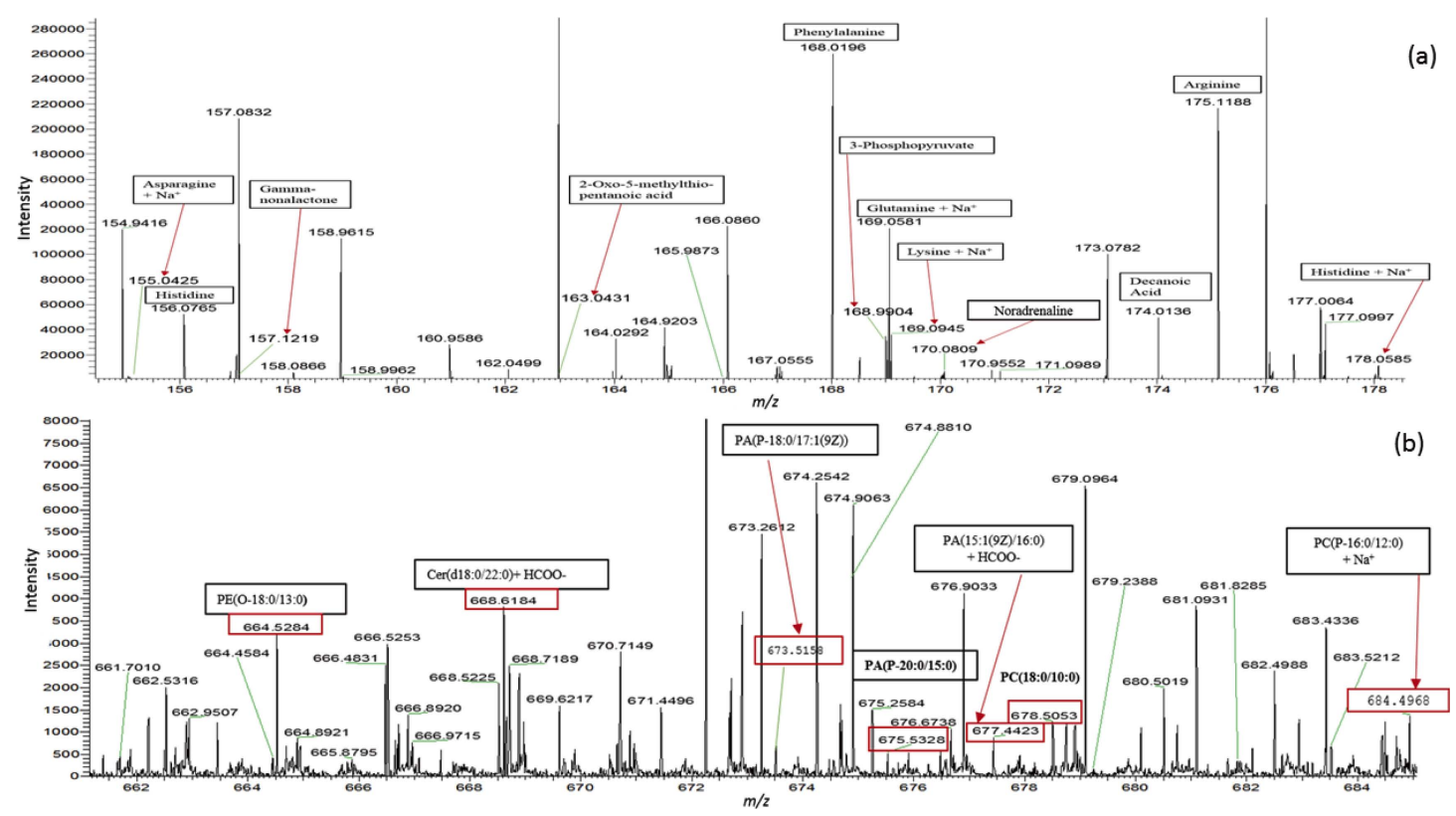

Fig. 4 Mass spectra showing (a) metabolite and (b) lipid peaks from a CTC with sonication in positive ion mode.

amino acids, including serine and glycine, as well as 5-hydroxylysine, the metabolite of lysine. Also, components specific to the cell membrane, such as 18:2 (15,16-DiHODE) and fatty acyl 5-oxo-7-decynoic acid (10:0), as well as numerous monoacylglycerides and triglycerides, were identified. Organelle specific compounds, such as deoxyuridine monophosphate (dUMP) (found only in the nucleus and mitochondria), were also detected. This result opens the door to "lipido-metabolomics" at the single-cell level and shows that this method has potential for diagnosis or health monitoring using only a drop of human blood.

The same method was applied to single CTC's. As shown in Fig. 4, super-sonication succeeded in detecting many metabolites (Fig. 4(a)) and lipids (Fig. 4(b)). Along with detected vital molecules such as amino acids, we found catecholamine metabolites such as noradrenaline $(\mathrm{m} / \mathrm{z}, 170.08)$, vanillactic acid $(\mathrm{m} / \mathrm{z}, 251.03)$ and homovanillic acid sulfate $(\mathrm{m} / \mathrm{z}$ 300.98) which were confirmed by MS/MS. In contrast, non-sonicated CTCs showed seven times fewer hits in the upper $\mathrm{m} / \mathrm{z}$ region, where lipids are found (310 lipids in sonicated vs. 42 lipids in nonsonicated) and the intensity of peaks was 20 - 100 fold less.

These detected metabolites will become the marker for the diagnosis of neuroblastoma. Usefulness of detected lipids is not clear at this stage. Drugs included in the patient's regimen, such as streptomycin $(\mathrm{m} / \mathrm{z}$ 582.27), were also detected, and confirmed by MS/MS in the sonicated samples, but were absent in non-sonicated cells. This infers that such single-cell analysis can be further used to monitor the concentration level drug delivery into the target cells. Since only a few CTCs can be collected, this single-cell analysis is very useful and we should continue to survey differences in molecular peaks during different stages and metastasis with many clinical cases.

However, we think the first base of exhaustive molecular analysis, "lipido-metabolomics," has been established by our development, in addition to current liquid biopsy methods of CTC's. We hope this method will be an alternative, powerful, and reliable diagnosis technique in the clinical field.

\section{Acknowledgements}

This study is approved by the Institutional Review Board of Hiroshima University (I-RINRI-Hi-No.20). We thank Thermo Fisher Scientific Inc., especially Dr. A. Makarov, for their support with scientific instruments, and Mrs. April Oga for assistance with writing this manuscript. This work was conducted under the Single Cell Science Project, and Integrated Lipidology Project in RIKEN and supported by the fund for the Development of Advanced Measurement and Analysis Systems (SENTAN), by Japan Agency for Medical Research and Development (AMED), and by Japan Science and Technology Agency (JST) in 2013 - 2014.

\section{References}

1. I. Cree, Pathogenesis, 2015, 2, 1.

2. N. Karachaliou1, C. Mayo-de-las-Casas, M. Molina-Vila, and R. Rosell, Ann. Transl. Med., 2015, 3, 36.

3. E. Heitzer, M. Auer, P. Ulz, J. Geigl, and M. Speicher, Genome Med., 2013, 5, 73.

4. P. Loo and T. Voet, Curr. Opin. Genetics Dev., 2014, 24, 82.

5. E. I. Galanzha and V. P. Zharov, Cancers, 2013, 5, 1692.

6. M. Yu, S. Stott, M. Toner, S. Maheswaran, and D. Haber, J. Cell. Biol., 2011, 192, 373.

7. D. Olmos, H. Arkenau, J. Ang, I. Ledaki, G. Attard, C. Carden, A. Reid, R. Herm, P. Fong, N. Oomen, R. Molife, D. Dearnaley, C. Parker, L. Terstappen, and J. Bono, Annals of Oncology, 2009, 20, 29.

8. J. Mateo, M. Gerlinger, D. Rodrigues, and J. de Bono, Genome Biology, 2014, 15, 448.

9. N. Tsuyama, H. Mizuno, E. Tokunaga, and T. Masujima, Anal. Sci., 2008, 24, 559.

10. H. Mizuno, N. Tsuyama, T. Harada, and T. Masujima, J. Mass Spectrum., 2008, 43, 1692.

11. T. Masujima, Anal. Sci., 2009, 25, 953.

12. T. Fujii, S. Matsuda, M. L. Tejedor, T. Esaki, I. Sakane, H. Mizuno, N. Tsuyama, and T. Masujima, Nat. Protoc., 2015, 10, 1445 . 